\title{
Synthesis of Thiol-Functionalized Ionic Liquids and Formation of Self-Assembled Monolayer on Gold Surfaces: Effects of Alkyl Group and Anion on the Surface Wettability
}

\author{
Bang Sook Lee and Sang-gi Lee* \\ Life Scrences Division. Korea Institate of Science and Technology, P. O. Box 131. Cheongryang. Seoul 130-650, Korea \\ Received Jily 21,2004
}

\begin{abstract}
Twenty four thiol-functionalized ionic liquids based on imidazolium cation, 1-(12-mercaptododecyl)-3alkylimidazolium salts. have been synthesized, and utilized to investigate the effects of alkyl-chain length and anion on the wetability of Au surfaces on the basis of self-assembled monolayers presenting [(CnSAMIM)X], where $n-1-6, \mathrm{X}-\mathrm{Br}$. B $\Gamma_{4}, \mathrm{PF}_{4}$ and $\mathrm{NT} \int_{2}$. Water wettabilities of the surfaces were measured as a water contact angle by contact angle goniometry. It was found that water wettability of the Au surfaces coated with imidazolium ions was largely dependent not only on counter anions but also on the length of alkyl chains. In the case of SAMs of $N$-alkylimidazolium ions having short Iength of $N$-alkyl chain $\left(\mathrm{C}_{1}-\mathrm{C}_{4}\right)$, anions played great role in determining water wettability of the surfaces.
\end{abstract}

Key Words : Thiol-functionalized ionic liquids. Self-assembled monolayer, Surface wettability, Anion effect

\section{Introduction}

Recently, a new class of liquid has emerged that consists entirely of ions, so-called ionic licjuids (ILs). They are made up of two components i.e. the anion and cation. As both anion and cation can be varied, these solvents can be designed for a particular set of properties such as solubility, density, refractive index, and viscosity. The favorable and readily tunable physicochemical properties of Il s, especially imidazolium cation-based Il s (Figure 1) have led to intense interest in these materials as alternatives to conventional organic solvents in a range of synthesis, catalysis, electrochemistry and liquid-liquid extractions. ${ }^{1-6}$ The burgeoning field of [l. research continues to demonstrate the potential for [I.s in variety of applications such as biopolymers, ${ }^{7.8}$ chemosensors for specific ions," formation of nanosized metal particles etc. ${ }^{10-12}$ Furthermore, their ionic nature makes them suitable for use as conductive media in lithium battery and solar cells. ${ }^{13-15}$

We reasoned that tunable physical properties of II ss could be transferred onto solid surfaces, whose properties. especially water wettability, could be adjusted by variation of the length of alkyl chain on cation and/or the anions. Controlling wettability of the solid surfaces by surface modification has intensively been studied due to many technologically important applications. ${ }^{16-18}$ Among the surface modification methods, the formation of self-assembled

$$
R^{1}-N \mathrm{X}^{+}-\mathrm{R}^{2} \quad \mathrm{X}=\mathrm{BF}_{4}, \mathrm{PF}_{6}, \mathrm{NTf}_{2} \text { etc. }
$$

Figure 1. 1.3-Dialkylimidarolium lonic I.iquids.

\footnotetext{
Conesponding Author. 'Tel: ·82-2-958-5163: lax: +82-2-958-5189:
} e-mail: sanggi ä kist.re.kr monolayers (SAMs) proved to be a simple and practical technique for controlling wettability. ${ }^{19-25}$ corrosion, ${ }^{26-28}$ and (bio)adhesion ${ }^{29-31}$ of solid surfaces. On the basis of SAMs, the wettability of solid surfaces could be controlled in various ways, i.e., environmental changes (solvents, ${ }^{35-37} \mathrm{pH}_{*}^{38}$ temperature, ${ }^{39-11}$ and surface pressure ${ }^{12}$ ) or external stimuli (i.e., light, ${ }^{23.24}$ charge, $^{25}$ and oxidation-reduction ${ }^{43}$ ). Our approach is a translation of the tunable water miscibility of [I.s in solution onto surfaces to control surface wettability. Recently, we have demonstrated that the wettability of SAMs presenting $N$-methyl imidazolium ions at the tail ends on gold ${ }^{\text {th }}$ or $\mathrm{Si} / \mathrm{SiO}_{2}$ surfaces ${ }^{15}$ could be controlled by anion exchange on surfaces ("anion-directed control"). Thus, the water contact angles of the SAMs terminating $N$-methylimidazolium ions were increased as change the hydrophilic anions such as $\mathrm{BF}_{4}{ }^{-}$to hydrophobic anions such as $\mathrm{NTf}_{2}^{-}$, which indicating clearly that the wettability of the surface can be tailored by changing the anion. Chujo of al also documented the surface of $\mathrm{Au}$ nanoparticles modified with ionic liquids, which allowed the their phase transfer through the anion exchange. ${ }^{16}$ The assortment of available and potential hydrophobic and hydrophilic II,s attests to their easily manipulated physical properties. Changing the length of alkyl groups and/or counter anions modulates the hydrophobicity of II $S$, and consequently water miscibility can be varied. Therefore, the combination of two components, alkyl chain and anion, will provide more diverse surfaces, which may control the wettability more finely. In this report, we synthesized various thiol-functionalized ionic liquids and formed SAMs terminating imidazolium moiety bearing different length of alky chain $\left(\mathrm{C}_{1}-\mathrm{C}_{6}\right)$ and anions $\left(\mathrm{Br}, \mathrm{BF}_{4}, \mathrm{PF}_{6}, \mathrm{NTf}_{2}\right)$, and investigated the effects of alkyl-chain length and anion on surface wettability on Au surfaces. 


\section{Results and Discussion}

In order to investigate the effects of alkyl-chain length and anion on the wettability of Au surfaces coated with imidazolium ion, twenty four thiol-functionalized ionic liquids bearing different length of $N$-alkyl groups (methyl, ethyl, n-propyl, n-butyl, n-pentyl and $n$-hexyl) and counter anions $\left(\mathrm{Br}, \mathrm{BF}_{4}, \mathrm{PF}_{6}\right.$ and $\mathrm{NTf_{2 }}$ ) representing hydrophilic and hydrophobic anions have been synthesized. The synthetic scheme for the thiol-functionalized imidazolium cationbased ionic liquids (3a-f, 4a-f, 5a-f and 6a-f) showed in Scheme1. Reaction of 1-alkyl imidazole la-f with 1,12dibromododecane in methylene chloride followed by nucleophilic substitution with potassium thioacetate could provide the imidazolium bromide $2 \mathbf{a}-\mathbf{f}$. The thioacethyl group of 2a-f was hydrolyzed with $\mathrm{NaOH}$ and acidified with $\mathrm{HBr}$ to give the corresponding thiol-functionalized imidazolium bromide salts $\mathbf{3 a - f}$. The anion exchange of $\mathbf{3} \mathbf{a}-\mathbf{f}$ with $\mathrm{NaBF}_{4}, \mathrm{NaPF}_{6}$ and $\mathrm{l}_{\mathrm{iN}} \mathrm{N}(\mathrm{Tf})_{2}$ in acetone provided the corresponding thiol-functionalized Il $s$ 4a-f, 5a-f and 6a-f, respectively. The imidazolium ion-terminated SAMs $[(\mathrm{CnSAMIM}) \mathrm{X}]\left[\mathrm{X}=\mathrm{Br}(7), \mathrm{BF}_{4}(\mathbf{8}), \mathrm{PF}_{6}(\mathbf{9}), \mathrm{NTf}_{2}(\mathbf{l 0})\right]$ having different alkyl groups and anions were formed by immersing freshly cleaned Au substrates in $1 \mathrm{mM}$ solution of thiol-functionalized ionic licjuids 3-6 in absolute ethanol for $3 \mathrm{~h}$.

Figure 2 shows the IR spectra in the $\mathrm{CH}$ stretching region for $\left[\left(\mathrm{C}_{1-6} \mathrm{SAMIM}\right) \mathrm{Br}\right]$. The characteristic $\mathrm{CH}_{2}$ asymmetric $\mathrm{CH}$ and symmetric $\mathrm{CH}$ stretching modes are observed at $2919-2922$ and $2846-2851 \mathrm{~cm}^{-1}$. The band at $2967-2985$ $\mathrm{cm}^{-1}$ is assigned to $\mathrm{CH}_{3}$ asymmetric in-plane $\mathrm{CH}$ stretching

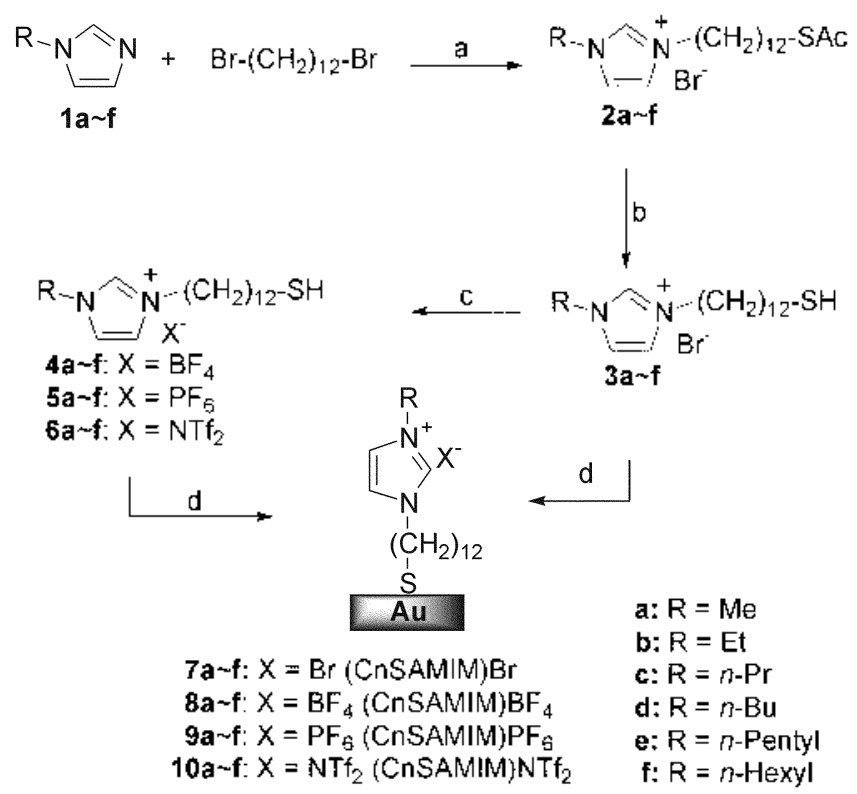

Sclome 1. Syntheses of thiol-functionalized ionic liquids (3a-f. 4a-f. 5a-f and 6a-f) having diflerent alkyl chains and anions. and the formation of self-assembled monolayers on Au surlaces. (a) (i) $\mathrm{CH}_{2} \mathrm{Cl} \mathrm{I}_{2}$. reflux. $10 \mathrm{~h}$. (ii) $\mathrm{KSCOCH}$. IH $\mathrm{H}_{3} \mathrm{O}$ (3/1. v/v). $\mathrm{NaOH} .0^{\circ} \mathrm{C}$. I h. then $\mathrm{HBr}$. (c) For 4: $\mathrm{NaBF}_{4}$; for 5 : $\mathrm{NaPF}_{0}$; for 6: L.jNTl. acetone. rt. 35 h. (d) Aul I m.M solution of thiol teminated ionic licpuids 3-6 in absolute ethanol for $3 \mathrm{~h}$. mode and the band at $2880-2894 \mathrm{~cm}^{-1}$ is assigned to the $\mathrm{CH}_{3}$ symmetric $\mathrm{CH}$ stretching mode. ${ }^{.77}$ These IR spectral data indicate that the monolayered films are well-ordered and closed-packed. The ellipsometric thicknesses of the SAMs 7a-7f (7a: $19 \AA, 7 \mathbf{b}: 21 \AA, 7 \mathrm{c}: 22 \AA, 7 \mathrm{~d}: 23 \AA, 7 \mathrm{e}: 24 \AA 7 \mathrm{f}$ $25 \dot{A})$ presenting $[(\mathrm{CnSAM} M) \mathrm{Br}$ ] also supported that the SAMs are packed densely.

The water wettabilities of the SAM surfaces were measured as a water contact angle by contact angle goniometry at $22{ }^{\circ} \mathrm{C}$ and $30 \%$ humidity. The data for the water contact angles of the SAMs (7-10) were obtained from mean value of three times measurements. Figure 3 showed the effects of alkyl chain-length on the wettability for each anion. The water contact angles of the SAMs presenting $\left[\left(\mathrm{C}_{11} \mathrm{SAMIM}\right) \mathrm{Br}\right]$ 7a-7 $\mathbf{f}$ were increased as increase the length of alkyl chain (7a: $23 \pm 1^{\circ}, 7 \mathbf{b}: 39 \pm 1^{\circ}, 7 \mathbf{c} ; 41 \pm 1^{\circ}, 7 \mathrm{~d}$ : $\left.43 \pm 1^{\circ}, 7 \mathrm{e} ; 62 \pm 1^{\circ}, 7 \mathrm{f} ; 63 \pm 1^{\circ}\right)$. The relatively larger change $\left(16^{\circ}\right)$ in the contact angle was observed by change the $N$ methyl, [(C,SAMIM)Br] (7a, $\left.23 \pm 1^{\circ}\right)$, to $N$-ethyl group. [(C $\mathrm{C}_{2}$ SAMIM $\left.) \mathrm{Br}\right]\left(7 \mathrm{~h}, 39 \pm 1^{\circ}\right)$. However, rather smaller changes in the contact angle (ca. $2^{\circ}$ ) have been observed between $7 \mathbf{b}-7 \mathbf{d}$ having $C_{2}-C_{4}$ alkyl chains. Dramatic alkyl chain-length effect was observed in SAM 7e [(C.SAMIM) $\mathrm{Br}$ ] having $\mathrm{C}_{5}$-hexyl chain. Compare to $7 \mathrm{~d}\left(43 \pm 1^{\circ}\right)$, about $20^{\circ}$ of contact angle was increased in $7 \mathrm{e}\left(62 \pm 1^{\circ}\right)$. Whereas there is no changes in the water contact angles of surfaces presenting [(C.5AMIM)Br] $\left(7 \mathrm{C}: 62 \pm 1^{\circ}\right)$ and [(C.6AMIM)Br] ( $7 \mathrm{f}: 63 \pm 1^{\circ}$ ). Interest finding is relatively smaller changes in the contact angles of the surfaces bearing relatively hydrophobic counter anions. In the cases of the SAMs bearing relatively hydrophilic counter anion $\mathrm{BF}_{4}, \mathbf{8 a - f}(\mathbf{8 a}$ : $35 \pm 1^{\circ}, 8 \mathrm{~b}: 43 \pm 1^{\circ}, 8 \mathrm{c}: 46 \pm 1^{\circ}, 8 \mathrm{~d}: 57 \pm 1^{\circ}, 8 \mathrm{e}: 80 \pm 1^{\circ} . \mathbf{8 f}$ $\left.84 \pm 1^{\circ}\right)$, the effects of alkyl chain-length were cuite similar with $7 \mathbf{a}-\mathbf{f}$ bearing a strong hydrophilic bromide anion, Thus, changing the length of alkyl chains from $\mathrm{C}_{4}(\mathbf{8 d})$ to $\mathrm{C}_{5}(\mathbf{8 e})$ increased about $20^{\circ}$ of the contact angles. In contrast, the SAM 9a-f bearing relatively hydrophobic counter anion $\mathrm{PF}_{4}$ showed smaller changes in the contact angles as increased the length of alkyl chains (9a: $52 \pm 1^{\circ}, 9 \mathbf{b}$ : $59 \pm 1^{\circ}, 9 \mathbf{c}$ : $59 \pm 1^{\circ}, 9 d: 69 \pm 1^{\circ}, 9 \mathrm{e}: 83 \pm 1^{\circ}, 9 f: 84 \pm 1^{\circ}$ ). Among the examined counter anions, as increase the length of alkyl chains, the most hydrophobic anion $\mathrm{N}^{\prime} \mathrm{ff}_{2}$ showed the smallest changes in the water contact angles of surfaces presenting $\left[\left(\mathrm{C}_{10} \mathrm{SAM}[\mathrm{M}) \mathrm{N}^{\prime} \mathrm{ff}_{2}\right]\right.$ (10a-f) (10a: $65 \pm \mathrm{I}^{\circ}, \mathbf{1 0 b}$ : $66 \pm 1^{\circ}, 10 \mathrm{c}: 68 \pm \mathrm{l}^{\circ}$, 10d: $78 \pm 1^{\circ}$, 10e: $82 \pm 1^{\circ}$, 10f: $85 \pm 1^{\circ}$ ). The different chain-length dependency of the surface wettability of the SAMs between the hydrophilic and hydrophobic anions may be related with the interactions between the anion, imidazolium cation and water molecules of the surface. The exact nature of the interactions remained to be solved. Nevertheless, these results clearly indicate that the wettability of the Au surfaces coated with alkylimidazolium ion is largely dependent on the length of alkyl chain attached onto the imidazolium moiety, i.e... the water contact angles of the surfaces increased as increase the length of alkyl chain.

We also found that the effects of counter anion on surface wettability were also dependent on the length of alkyl 

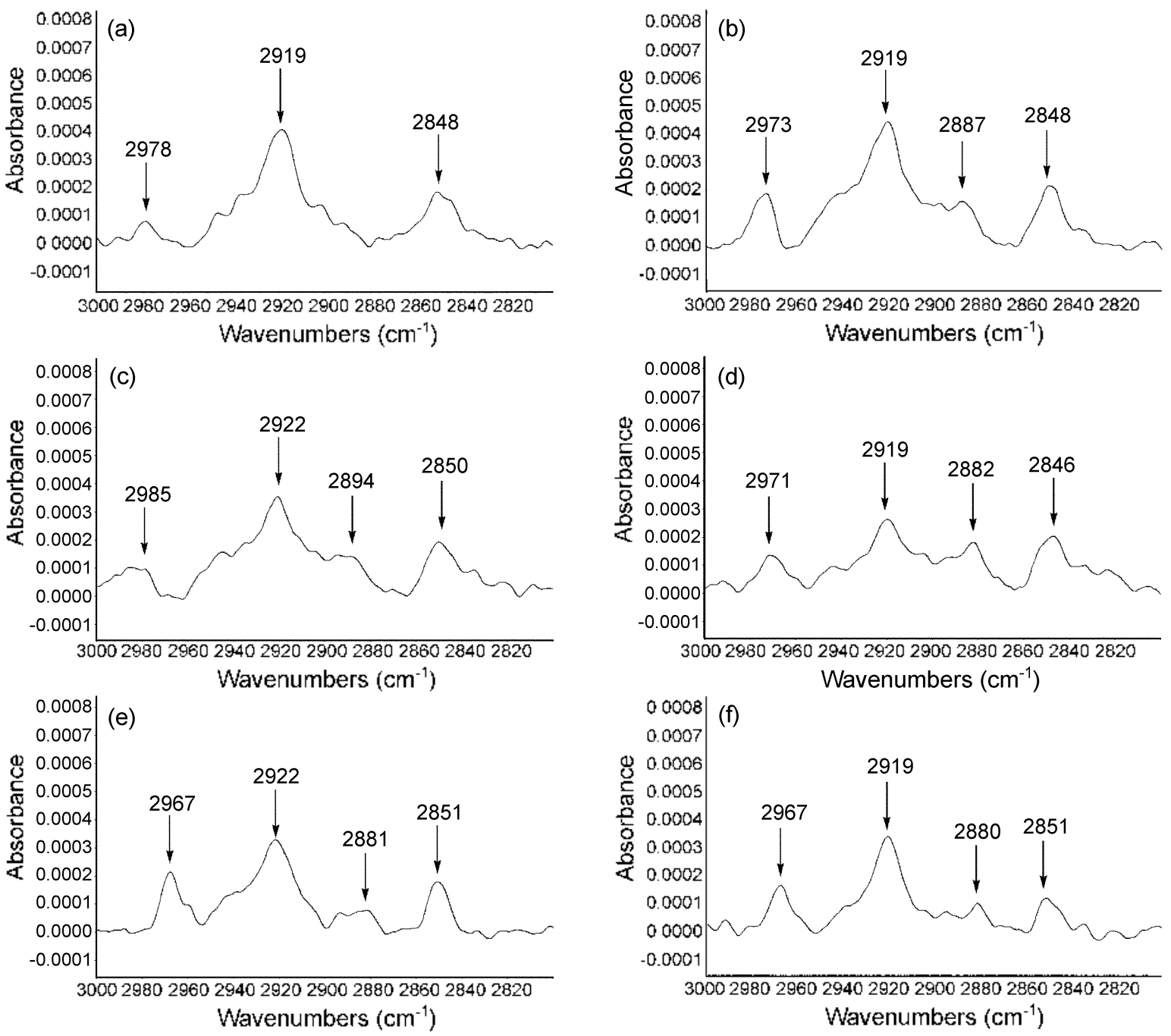

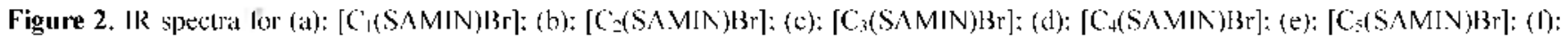
$|\mathrm{C}(\mathrm{S} \Lambda \mathrm{MIN}) \mathrm{Br}|$.

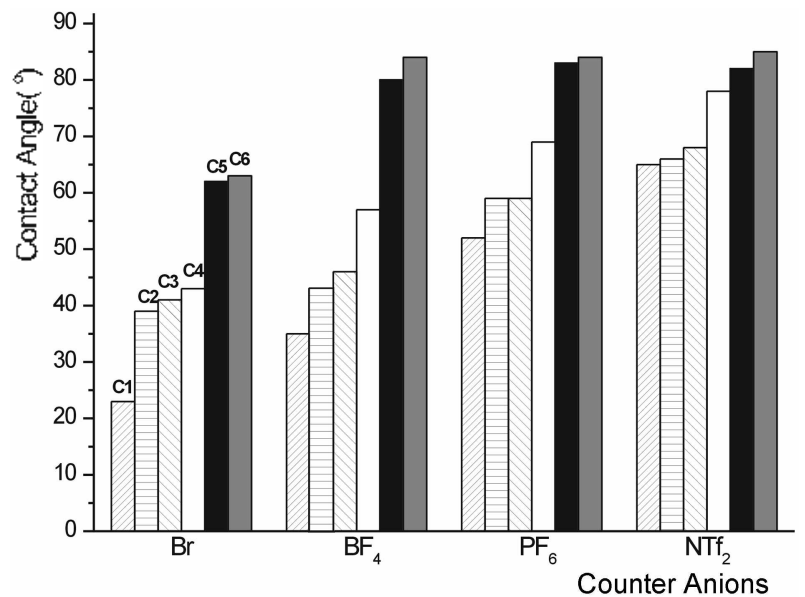

Figure 3. Graph of water contact angles versus length of alkyl chain for each counter anion.

chains. Figure 4 is another data graphic showing more clearly the effects of counter anion on the water contact angles for each SAMs having different alkyl chain length. As

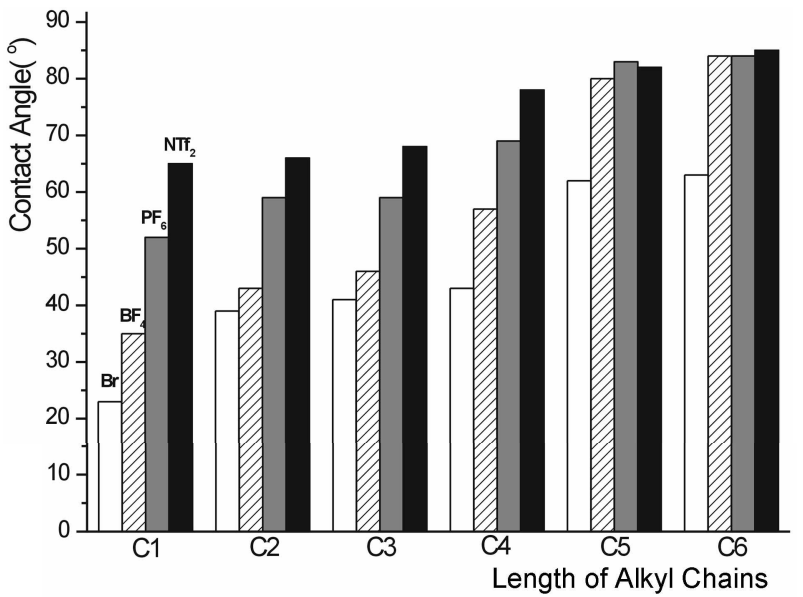

Figure 4. Graph of water contact angles versus counter anion for each alkyl chain.

we observed previously, the water contact angles of the SAMs presenting $[(\mathrm{C}, \mathrm{SAMIM}) \mathrm{X}]$ were systematically increased as change the counter anion from $\mathrm{Br}\left(7 \mathbf{a}: 23 \pm 1^{\circ}\right)$ to $\mathrm{BF}_{+}(8 \mathbf{a}$ : 
$\left.35 \pm 1^{\circ}\right), \mathrm{PF}_{6}\left(9 \mathrm{a}: 52 \pm 1^{\circ}\right)$ and $\mathrm{NTf}_{2}\left(10 \mathrm{a}: 65 \pm 1^{\circ}\right) .{ }^{H}$ However, in the cases of the SAMs bearing $\mathrm{C}_{2}$ or $\mathrm{C}_{3}$ alkyl chains. changes in contact angles were distinguished by the nature of the counter anions. Thus, relatively smaller changes in the contact angles of the surfaces bearing hydrophilic counter anions $\mathrm{Br}$ and $\mathrm{BF}_{4}\left(7 \mathrm{~b}: 39 \pm 1^{\circ}\right.$ vs. $8 \mathrm{~b}: 43 \pm 1^{\circ}: 7 \mathrm{c}: 41 \pm 1^{\circ}$ vs. $8 \mathrm{c}: 46 \pm 1^{\circ}$ ) were observed compared to those bearing hydrophobic counter anions $\mathrm{PF}_{6}$ and $\mathrm{NTf}_{2}\left(9 \mathrm{~b}\right.$ : $59 \pm \mathrm{l}^{\circ} \mathrm{v}$. 10b: $66 \pm 1^{\circ} ; 9 \mathrm{c}: 59 \pm 1^{\circ}$ ws: 10c: $68 \pm 1^{\circ}$ ). These results may suggest that the wettability of the SAM surfaces coated with $\mathrm{C}_{1}-\mathrm{C}_{3}$-alkylimidazolium ions controlled mainly by anion property. In case of the $C_{4}-S A M$ presenting [( $C_{4}$ SAMIM)X]. both alkyl group and counter anion contributed almost equally to the wettability of the surfaces. and the contact angles were changed relatively regularly by changing the counter anions (7d: $43 \pm 1^{\circ} .8 d$ : $57 \pm 1^{\circ}, 9 d: 69 \pm 1^{\circ}$. 10d: $78 \pm 1^{\circ}$ ). However, the anions did not siguificantly affect the surface wettability of the $\mathrm{C}_{5}$ - and $\mathrm{C}_{6}$-SAMs presenting $\left[\left(C_{S} S A M M M\right) X\right]$ and $\left[\left(C_{6} S A M M M\right) X\right]$. respectively, and the water wettabilities of the surfaces were mainly controlled by the alkyl groups. Little changes in contact angles of these surfaces imply that anions may be embedded in the relatively long pentyl or hexyl chains and in close contact with imidazolium cation. Therefore. effective "aniondirected" control of water wettability of Au surfaces coated with SAMs terminating in imidazolium ions could be possible only when the alkyl groups attached on the inidazolium moiety have less than four carbon atoms. Nevertheless. a strong hydrophilic bromide anion still affected the surface wettability. and thus. much smaller water contact angles were observed in $\left[\left(\mathrm{C}_{5.6} \mathrm{SAMIM}\right) \mathrm{Br}\right]$ compared to those observed in $\left[\left(\mathrm{C}_{\Sigma .6} \mathrm{SAMIM}\right) \mathrm{X}\right]\left(\mathrm{X}=\mathrm{BF}_{4}\right.$. $\mathrm{PF}_{6}$ and $\mathrm{NTf}_{2}$ ) bearing other counter anion. These anion effects are quite different with those observed on $\mathrm{Si}_{2} / \mathrm{SiO}_{2}$ surfaces. where counter anions did not effect at all the wettability of surfaces coated with $n$-butylimidazolium ions. Unfortunately, it is hard to explain at present time why $\mathrm{All}$ and $\mathrm{Si} / \mathrm{SiO}_{2}$ surfaces differently response to the alkyl-chain length and counter anion on the water wettability.

In summary, modulation of surface properties has important implications in both fundamental and technological advances. We reasoned that tunable water-miscibility of ILs could be transferred onto solid surface wettability, which could be adjusted by variation the length of alkyl chain on cation and/or anions. We designed and synthesized various kinds of thiol-functiolized ionic liquids having different length of alkyl chains and counter anions. and formed SAMs on Au surfaces with the ain of examining the effects of alkyl-chain length and counter anion on surface wettability. We found that the wettability of the Au surfaces coated with imidazolium ions were dependent not only on the length of alkyl-chain but also on counter anions.

\section{Experimantal Section}

General methods. NMR spectra were obtained on a Brikker $300 \mathrm{MHz} .{ }^{l} \mathrm{H}$ NMR spectra were referenced to tetramethylsilane in $\mathrm{CDCl}_{3}:{ }^{13} \mathrm{C}$ NMR spectra were referenced to residual solvent. All reagents were used as received. IMetlyylimidazole and 1,12-dibromododecane. $\mathrm{NaBF}_{4}, \mathrm{NaPF}_{6}$. $\mathrm{LiN}\left(\mathrm{SO}_{2} \mathrm{CF}_{3}\right)$ were obtained from Sigma-Aldrich Korea or Acros Organics unless othenvise specified. All organic solvents were dried under standard purification conditions and distilled water was used. Polarized infrared external reflectance spectroscopy (PIERS) spectra were obtained in a single reflection mode using $\mathrm{N}_{2}$-purged Thermo Nicolet Nexus Fourier Transform infrared spectrometer. The $p$ polarized light was incident at $80^{\circ}$ relative to surface normal of the substrate and a narrow band mercury-cadmiumtelluride (MCT) detector was used. We averaged 1024 scans to yield the spectrum at a resolution of $2 \mathrm{~cm}^{-1}$. The sample compartment was purged with dry and $\mathrm{CO}_{2}$ free $\mathrm{N}_{2}$. An ellipsometer (Gaertner Lll6s) equipped with a He-Ne Laser $(632.8 \mathrm{rm})$ was used to determine the thickness of the monolayers. A contact angle instrument (Phoenix 300 , Surface Electro Optics Co. Ltd., Korea) was used to determine the water contact angles of the monolayers and the water contact angles were measured at $22^{\circ} \mathrm{C}$ and under $30 \%$ humidity. The Au substrates were prepared by thermal evaporation of $100 \mathrm{~nm}$ of $\mathrm{Au}$ onto polished $\mathrm{Si}(100)$ with a 20 $\mathrm{nm}$ Ti adhesion layer and cut into $1 \mathrm{~cm}^{2}$ pieces.

General synthetic procedure for 1-[12-(thioacetyl) dodecyl]-3-alkylimidazolium bromide (2a-f). A solution of 1 -alkylimidazole $(25 \mathrm{mmol})$ in metlyy lene chloride $(10 \mathrm{~mL})$ was added to a solution of 1,12-dibromododecane $(25 \mathrm{~g} .78$ mumol) at room temperature. The reaction mixture was heated at reflux for $10 \mathrm{~h}$. After evaporation of the solvent, the unreacted 1.12-dibromododecane was washed out thoroughly with hexane. The resulting solid was purified by silica column chromatograply $\left(\mathrm{CH}_{2} \mathrm{Cl}_{2} / \mathrm{MeOH}=7 / 1\right)$ to give 1-(12-bromododecyl)-3-alkylimidazolium bromide. To a solution of 1-(12-bromododecyl)-3-alkylimidazolium bromide $(6.2 \mathrm{mmol})$ in dry THF $(10 \mathrm{~mL})$ was added potassium thioacetate $(0.73 \mathrm{~g}, 6.2 \mathrm{mmol})$. After reflux for 6 h. the reaction mixture was cooled to room temperature. and the precipitate appeared was filtered off.s The filtrate was evaporated to remove solvent. and the residue was purified by silica colunn chromatography $\left(\mathrm{CH}_{3} \mathrm{Cl}_{2} / \mathrm{MeOH}=7 / 1\right)$ to give $1-[12$-(thioacetyl) dodecyl]-3-alkylimidazolium bromides 2a-f.

2a: ${ }^{l} \mathrm{H}$ NMR ( $\left.\mathrm{CDCl}_{3}\right) \delta 1.30-1.21(\mathrm{~m} .16 \mathrm{H}) .1 .54(\mathrm{~m}, 2 \mathrm{H})$. 1.89 (m. 2H). 2.29 (s. 3H). 2.82 (t. $J=7.33$ Hz. $2 \mathrm{H}$ ). 4.11 (s. $3 \mathrm{H}$ ). 4.29 (t. $J=7.42 \mathrm{~Hz} .2 \mathrm{H}$ ). 7.38 (s. 1H.). 7.54 (s. $1 \mathrm{H}$ ) 10.36 (s. $1 \mathrm{H}):{ }^{13} \mathrm{C}$ NMR $\left(\mathrm{CDCl}_{2}\right) \delta 26.1 .28 .7,28.9,29.0$. 29.2. 29.3, 29.4, 30.2. 30.5, 36.7, 50.1. 121.7, 122.8. 137.9. 196.1.

2b: ${ }^{1} \mathrm{H}$ NMR $\left(300 \mathrm{MHz} . \mathrm{CDCl}_{3}\right) \delta 0.96($ t. $J=7.38 \mathrm{~Hz}$. 3H. $\left.\mathrm{CH}_{3}\right), 1.29-1.20\left(\mathrm{~m}, 16 \mathrm{H} . \mathrm{CH}_{2}\right), 1.52\left(\mathrm{~m}, 2 \mathrm{H} . \mathrm{CH}_{2}\right) .1 .95$ (m. $2 \mathrm{H}, \mathrm{CH}_{2}$ ), 2.29 (s. $\left.3 \mathrm{H} . \mathrm{CH}_{3}\right), 2.82$ (t. $J=7.29 \mathrm{~Hz}, 2 \mathrm{H}$. $\mathrm{CH}_{2}$ ) 4.32 (t. $J=7.29 \mathrm{~Hz}, 4 \mathrm{H}, \mathrm{CH}_{2}$ ). 7.44 (t. $J=1.77 \mathrm{~Hz}$. 1H. Imid). 7.53 (t. $J=1.77 \mathrm{~Hz}, 1 \mathrm{H}$. Imid). 10.50 (s. $1 \mathrm{H}$. Imid): ${ }^{13} \mathrm{C}$ NMR $\left(75.5 \mathrm{MHz}, \mathrm{CDCl}_{3}\right) \delta 26.2,28.1,28.6$, 28.9. 29.2. 29.3, 30.2, 32.7, 34.1. 36.7. 50.2, 121.7. 123.4. 137.7. 196.1 
2c: ${ }^{~} \mathrm{H}$ NMR $\left(300 \mathrm{MHz}, \mathrm{CDCl}_{\mathfrak{j}}\right) \delta 0.97(\mathrm{t}, J=7.32 \mathrm{~Hz}$. $\left.3 \mathrm{H}, \mathrm{CH}_{3}\right), 1.43-1.24\left(\mathrm{~m}, 18 \mathrm{H}, \mathrm{CH}_{2}\right) .1 .56\left(\mathrm{~m}, 2 \mathrm{H}, \mathrm{CH}_{2}\right) .2 .32$ $\left(\mathrm{s}, 3 \mathrm{H}, \mathrm{CH}_{3}\right), 2.86$ (t. $\left.J=7.32 \mathrm{~Hz} .2 \mathrm{H}, \mathrm{CH}_{2}\right), 4.38(\mathrm{~m} . J=$ $\left.7.53 \mathrm{~Hz} .4 \mathrm{H} . \mathrm{CH}_{3}\right), 7.48$ (s, 1H, Imid), 7.54 (s, $1 \mathrm{H}$, Imid). 10.57 (s. $1 \mathrm{H}$. Imid) ${ }^{13} \mathrm{C}$ NMR $\left(75.5 \mathrm{MHz} . \mathrm{CDCl}_{\hat{3}}\right) \delta 10.62$. $24.60,26.20 .28 .85,28.94,29.01 .29 .21,29.28,29.33$. $29.35,30.23 .30 .53,32.09,49.71 .49 .90,121.50 .121 .70$. 137.11, 196.20.

2d: ${ }^{1} \mathrm{H}$ NMR $\left(300 \mathrm{MHz}, \mathrm{CDCl}_{3}\right) \delta 0.97(\mathrm{t}, J=7.34 \mathrm{~Hz}$. $\left.3 \mathrm{H}, \mathrm{CH}_{3}\right), 1.44-1.25\left(\mathrm{~m}, 20 \mathrm{H}, \mathrm{CH}_{2}\right) .1 .56\left(\mathrm{~m}, 2 \mathrm{H}, \mathrm{CH}_{2}\right) .1 .93$ (m. $\left.2 \mathrm{H}, \mathrm{CH}_{2}\right), 2.32\left(\mathrm{~s}, 3 \mathrm{H} . \mathrm{CH}_{3}\right), 4.86(\mathrm{t}, J=7.34 \mathrm{~Hz}, 2 \mathrm{H}$. $\mathrm{CH}_{3}$ ). 4.38 (q. $J=7.40 \mathrm{~Hz}, 2 \mathrm{H} . \mathrm{CH}_{2}$ ). 7.38 (s. IH. Imid). 7.42 (s. $1 \mathrm{H}$, Imid). 10.68 (s. $1 \mathrm{H}$, Imid): ${ }^{13} \mathrm{C}$ NMR $(75.5$ $\mathrm{MHz} . \mathrm{CDCl}_{3}$ ) $\delta 13.36 .19 .36,26.12,28.66 .28 .85,28.94$. $29.01,29.21$. 29.28, 29.33, 29.35. 30.23, 30.53, 32.09 . $49.71,29.99,121.52 .122 .02,137.12,195.97$.

2e: ${ }^{1} \mathrm{H}$ NMR $\left(300 \mathrm{MHz}, \mathrm{CDCl}_{\hat{j}}\right) \delta 0.89(\mathrm{t}, J=6.63 .6 .93$ $\mathrm{Hz}, 3 \mathrm{H}, \mathrm{CH}_{3}$ ). $1.35-1.24$ (m, 22H. CH$), 1.54$ (m. $2 \mathrm{H} . \mathrm{CH}_{2}$ ). 1.95 (n. $\left.2 \mathrm{H} . \mathrm{CH}_{2}\right), 2.32$ (s. $\left.3 \mathrm{H} . \mathrm{CH}_{3}\right), 2.86\left(\mathrm{t}_{\mathrm{t}} J=7.29,7.35\right.$ $\left.\mathrm{Hz}, 2 \mathrm{H} . \mathrm{CH}_{2}\right), 4.38\left(\mathrm{~m} .4 \mathrm{H} . \mathrm{CH}_{2}\right) .7 .65(\mathrm{t} . J=1.7 \mathrm{I} \mathrm{Hz}, \mathrm{lH}$. Imid). 7.61 (t, $J=1.71 \mathrm{~Hz}, \mathrm{lH}$. Inid), 10.45 (s, 1H, Imid): ${ }^{13} \mathrm{C}$ NMR $\left(75.5 \mathrm{MHz} . \mathrm{CDCl}_{\hat{3}}\right) \delta 13.54 .21 .75 .25 .92 .27 .92$. $28.46,28.68 .28 .75,28.81,29.04 .29 .08,29.10,29.14$. $29.70,30.05,30.36,49.68,49.72 .121 .96,122.10,136.51$. 195.80 .

2f: ${ }^{~} \mathrm{H}$ NMR $\left(300 \mathrm{MHz}, \mathrm{CDCl}_{\hat{j}}\right) \delta 0.87$ (t. $J=6.90 \mathrm{~Hz} .3 \mathrm{H}$. $\left.\mathrm{CH}_{3}\right) .1 .31-1.24\left(\mathrm{~m}, 24 \mathrm{H}, \mathrm{CH}_{2}\right), 1.55\left(\mathrm{~m} .2 \mathrm{H} . \mathrm{CH}_{3}\right), 1.92(\mathrm{~m}$. $\left.4 \mathrm{H}, \mathrm{CH}_{2}\right), 2.32$ (s. $\left.3 \mathrm{H}_{1} \mathrm{CH}_{3}\right), 2.86\left(\mathrm{t}, J=7.32 \mathrm{~Hz}, 2 \mathrm{H}_{2} \mathrm{CH}_{2}\right.$ ) $4.37\left(\mathrm{~m}, 4 \mathrm{H}, \mathrm{CH}_{2}\right) .7 .58(\mathrm{t}, J=1.68 \mathrm{~Hz}$. IH. Imid). $7.6 \mathrm{l}(\mathrm{t} . J$ $=1.63 \mathrm{~Hz} .1 \mathrm{H}$, Imid), 10.44 (s. $1 \mathrm{H}$, Imid) ${ }^{13} \mathrm{C}$ NMR $(75.5$ $\left.\mathrm{MHz} . \mathrm{CDCl}_{3}\right) \delta 13.66 .22 .10,25.58,25.96 .28 .49,28.71$. $28.78,28.84 .29 .10,29.11 .29 .14,29.17,30.00,30.07$. $30.79,49.75,121.94 .122 .02,136.92,195.90$.

General synthetic procedure for 1-(12-mercaptododecyl)3-alkylimidazolium bromide (3a-3f). To a solution of 1 [12-(S-acetyl)dodecyl]-3-alkylimidazolium bromide $(0.247$ mmol) in EtOH ( $3 \mathrm{~mL}$ ) was added a solution of $\mathrm{NaOH}(0.01$ g. $0.247 \mathrm{mmol})$ in $\mathrm{H}_{2} \mathrm{O}(1.0 \mathrm{~mL})$ at $0{ }^{\circ} \mathrm{C}$. The reaction mixture was stirred at the same temperature, and then acidified with $2 \mathrm{~N} \mathrm{HBr}$ until $\mathrm{pH}=2$. The organic materials were extracted with $\mathrm{CH}_{2} \mathrm{Cl}_{2}$. and dried with $\mathrm{MgSO}_{4}$ evaporated to give product 1-(12-mercaptododecyl)-3alkylinidazolium bromide $3 \mathrm{a}-\mathrm{f}$.

3a: ${ }^{1} \mathrm{H} \mathrm{NMR}\left(\mathrm{CDCl}_{\hat{3}}\right) \delta 1.34-1.25(\mathrm{~m} .17 \mathrm{H}) .1 .36(\mathrm{~m}, 2 \mathrm{H})$. $1.58(\mathrm{~m}, 2 \mathrm{H}), 1.91(\mathrm{~m} .2 \mathrm{H}), 2.51$ (q. $J=7.42 \mathrm{~Hz} .2 \mathrm{H}), 4.13$ (s. $3 \mathrm{H}) .4 .32$ (t. $J=7.49 \mathrm{~Hz}) .7 .33$ (s. IH). 7.45 (s, lH). $10.53(\mathrm{~s}, 1 \mathrm{H}):{ }^{13} \mathrm{C}$ NMR $\left(\mathrm{CDCl}_{3}\right) \delta 24.6,26.2 .28 .3 .28 .9$. $29.2,29.4 .30 .2,33.9 .36 .9,50.1 .121 .6 .123 .4,137.9$.

3b: ${ }^{\mathrm{H}} \mathrm{HMR}\left(300 \mathrm{MHz}, \mathrm{CDCl}_{3}\right) \delta 0.97(\mathrm{t}, J=7.38 \mathrm{~Hz} .3 \mathrm{H}$. $\left.\mathrm{CH}_{3}\right) .1 .31-1.2+\left(\mathrm{m} .16 \mathrm{H}_{2} \mathrm{CH}_{2}\right.$ and $\left.\mathrm{SH}\right), 1.56\left(\mathrm{~m}, 2 \mathrm{H} . \mathrm{CH}_{2}\right)$. $1.88\left(\mathrm{~m} .2 \mathrm{H}_{2} \mathrm{CH}_{2}\right), 2.86\left(\mathrm{t}, J=7.33 \mathrm{~Hz} .2 \mathrm{H}, \mathrm{CH}_{2}\right) .4 .20(\mathrm{~m} . J$ $=1.72 \mathrm{~Hz}, 1 \mathrm{H}$. Imid). 7.38 (t. $J=1.72 \mathrm{~Hz}, 1 \mathrm{H}$, Imid), 8.91 (s. IH. Imid); ${ }^{13} \mathrm{C}$ NMR $\left(75.5 \mathrm{MHz} . \mathrm{CDCl}_{3}\right) \delta 23.43,26.15$. $28.75,28.89 .29 .07,29.10,29.30 .29 .37,29.43,29.45$. $30.17,30.62,50.12,51.52,122.07 .122 .23 .135 .90$.

3c: ${ }^{l} \mathrm{H}$ NMR $\left(300 \mathrm{MHz} . \mathrm{CDCl}_{3}\right) \delta 1.37\left(\mathrm{~m}, 19 \mathrm{H} . \mathrm{CH}_{2}\right.$ and $\mathrm{SH}), 1.65-1.56$ (n. $5 \mathrm{H} . \mathrm{CH}_{2}$ and $\mathrm{CH}_{3}$ ). 1.93 (n. $2 \mathrm{H}$. $\mathrm{CH}_{2}$ ).
2.53 (q. $\left.J=7.47 \mathrm{~Hz}, 2 \mathrm{H} . \mathrm{CH}_{2}\right), 4.35(\mathrm{~m} . J=7.47 \mathrm{~Hz} .2 \mathrm{H}$, $\left.\mathrm{CH}_{3}\right) .7 .38$ (t. $J=1.65 \mathrm{~Hz} .1 \mathrm{H}$. Imid). $7.54(\mathrm{~s}, J=1.65 \mathrm{~Hz}$, 1H. Imid). 10.64 (s. 1H. Imid); ${ }^{13} \mathrm{C}$ NMR $(75.5 \mathrm{MHz}$. $\left.\mathrm{CDCl}_{3}\right) \delta 15.62,24.59,26.20,28.26 .28 .91 .28 .96 .29 .26$. 29.36, 30.27. 33.94. 45.25. 50.10. 121.55, 121.69, 137.10.

3d: ${ }^{1} \mathrm{H}$ NMR $\left(300 \mathrm{MHz} . \mathrm{CDCl}_{3}\right) \delta 0.98(\mathrm{t}, J=7.34 \mathrm{~Hz}$, $3 \mathrm{H} . \mathrm{CH}_{2}$ ). $1.59-1.32$ (m. $21 \mathrm{H} . \mathrm{CH}_{2}$ and $\mathrm{SH}$ ). 1.60 (m. $2 \mathrm{H}$, $\left.\mathrm{CH}_{2}\right), 1.92\left(\mathrm{~m}, 2 \mathrm{H}, \mathrm{CH}_{2}\right) .2 .57$ (m. $\left.2 \mathrm{H} . \mathrm{CH}_{2}\right), 4.37$ (q, $J=$ $7.40 \mathrm{~Hz}, 2 \mathrm{H}, \mathrm{CH}_{2}$ ), 7.27 (s. IH. Imid), 7.29 (s, lH. Imid), 10.81 (s, lH. Imid): ${ }^{13} \mathrm{C}$ NMR (75.5 MHz. $\left.\mathrm{CDCl}_{3}\right) \delta 13.85$, $22.31,24.59,25.81$. 26.12. 28.27, 28.92. 28.96. 29.28, $29.35,30.23,30.53 .32 .09,49.71,29.99,121.70,122.08$. 137.10

3e: ${ }^{1} \mathrm{H}$ NMR $\left(300 \mathrm{MHz}, \mathrm{CDCl}_{3}\right) \delta 0.84(\mathrm{t}, J=6.8 \mathrm{~Hz}, 3 \mathrm{H}$, $\left.\mathrm{CH}_{2}\right), 1.32-1.20\left(\mathrm{~m}, 23 \mathrm{H}, \mathrm{CH}_{2}\right.$ and $\left.\mathrm{SH}\right), 1.55\left(\mathrm{~m}, 2 \mathrm{H} . \mathrm{CH}_{2}\right)$, $1.88\left(\mathrm{~m} .2 \mathrm{H}, \mathrm{CH}_{2}\right), 2.46$ (t. $\left.J=7.32 \mathrm{~Hz} .2 \mathrm{H}, \mathrm{CH}_{2}\right), 4.30(\mathrm{~m}$, $\left.4 \mathrm{H} . \mathrm{CH}_{2}\right), 7.45(\mathrm{~s}, J=6.8 \mathrm{~Hz}, 1 \mathrm{H}$, Imid). $7.47(\mathrm{~s}, J=1.62$ Hz. 1H. Imid). 10.26 (s. $1 \mathrm{H}$, Imid): ${ }^{13} \mathrm{C} \mathrm{NMR}\left(\mathrm{CDCl}_{3}\right) \delta$ $13.67,21.88,24.46 .26 .06 .28 .06,28.15 .28 .81$. 28.84. $29.17,29.25,29.28 .29 .80 .30 .15,33.82 .49 .87 .49 .92$, $50.16,121.82,122.01 .136 .71$

3f: ${ }^{\mathrm{i}} \mathrm{H}$ NMR $\left(300 \mathrm{MHz}, \mathrm{CDCl}_{3}\right) \delta 0.88(\mathrm{t}, J=6.72 \mathrm{~Hz} .3 \mathrm{H}$, $\left.\mathrm{CH}_{3}\right), 1.36-1.25\left(\mathrm{~m}, 24 \mathrm{H}, \mathrm{CH}_{2}\right.$ and $\left.\mathrm{SH}\right), 1.61\left(\mathrm{~m}, 2 \mathrm{H} . \mathrm{CH}_{3}\right)$, $1.93\left(\mathrm{~m}_{2}, 4 \mathrm{H}, \mathrm{CH}_{2}\right) .2 .52\left(\mathrm{q}, J=7.32 \mathrm{~Hz} .2 \mathrm{H} . \mathrm{CH}_{2}\right) .4 .37(\mathrm{~m}$, $4 \mathrm{H} . \mathrm{CH}_{2}$ ). 7.28 (s. 1H. Imid), 7.32 (s, lH. Imid). 10.77 (s. 1H. Imid): ${ }^{13} \mathrm{C} \mathrm{NMR}\left(\mathrm{CDCl}_{3}\right) \delta 15.60 .22 .22 .25 .40,25.98$, $28.55,28.72,28.80$. 28.90. 29.22, 29.25. 29.15. 29.18, $23.10,30.11 .30 .80 .49 .85 .121 .85,122.02,136.59$.

Synthesis of 1-(12-mercaptododecyl)-3-alkylimidazolium tetrafluoroborate (4a-4f), hexafluorophosphate (5a-5f) and bis( $N$-trifluoromethansulfonate) (6a-6f). To a suspension of 1-(12-mercaptododecyl)-3-alkylimidazolium bromide (3) $(0.49 \mathrm{mmol})$ in acetone $(7 \mathrm{~mL})$ was added $\mathrm{NaBF}_{4}(0.64$ mumol) for 4 or $\mathrm{NaPF}_{6}(0.64 \mathrm{mmol})$ for 5 and $\mathrm{Li}\left(\mathrm{NTf}_{2}\right)$ for 6 . The mixture was stirred at room temperature for $35 \mathrm{~h}$. The solid was filtered off through Celite, and the filtrate was evaporated to remove solvent. The residue was purified by silica column cluromatography to give the anion-exchanged products $4 \mathrm{a}-4 \mathrm{f}$. $5 \mathrm{a}-\mathbf{5 f}$ and $\mathbf{6 a}-\mathbf{6 f}$.

ta: ${ }^{1} \mathrm{H}$ NMR $\left(\mathrm{CDCl}_{3}\right) \delta 1.36-1.26(\mathrm{~m} .17 \mathrm{H}) .1 .61(\mathrm{~m} .2 \mathrm{H})$, 1.89 (m. $2 \mathrm{H}$ ). 2.53 (q. $J=7.39,2 \mathrm{H}) .3 .96$ (s. $3 \mathrm{H}) .4 .17$ (t. $J=$ $7.44 \mathrm{~Hz}, 2 \mathrm{H}), 7.28$ (s. $1 \mathrm{H}$ ), 7.36 (s. $1 \mathrm{H}$ ). 8.82 (s. $1 \mathrm{H}$ ): ${ }^{13} \mathrm{C}$ NMR $\left(\mathrm{CDCl}_{3}\right) \delta 26.0,28.7,28.8$. 29.6. 29.0. 29.2, 29.3, 29.4. 29.8, 30.5. 36.0, 50.0, 122.0. 123.6. 135.6 .

tb: ${ }^{1} \mathrm{H}$ NMR $\left(300 \mathrm{MHz} . \mathrm{CDCl}_{3}\right) \delta 0.97(\mathrm{t}, J=7.38 \mathrm{~Hz}$, 3H. $\mathrm{CH}_{3}$ ). 1.31-1.24 (m. 16H. $\mathrm{CH}_{2}$ and $\mathrm{SH}$ ). $1.56(\mathrm{~m} .2 \mathrm{H}$, $\left.\mathrm{CH}_{2}\right), 1.88\left(\mathrm{~m} .2 \mathrm{H}, \mathrm{CH}_{2}\right) .2 .86\left(\mathrm{t}, J=7.33 \mathrm{~Hz}, 2 \mathrm{H} . \mathrm{CH}_{2}\right)$, 4.20 (m. $J=1.72 \mathrm{~Hz}, 1 \mathrm{H}$. Imid), 7.38 (t. $J=1.72 \mathrm{~Hz} .1 \mathrm{H}$, Imid), 8.91 (s, $1 \mathrm{H}$, Imid); ${ }^{13} \mathrm{C}$ NMR $\left(75.5 \mathrm{MHz} . \mathrm{CDCl}_{3}\right) \delta$ $23.43,26.15,28.75 .28 .89 .29 .07,29.10$. 29.30. 29.37. $29.43,29.45,30.17,30.62,50.12,51.52$. 122.07, 122.23, 135.90.

tc. ${ }^{1} \mathrm{H}$ NMR $\left(300 \mathrm{MHz}, \mathrm{CDCl}_{3}\right) \delta 1.31 \cdot 1.25\left(\mathrm{~m} .19 \mathrm{H} . \mathrm{CH}_{2}\right.$ and $\mathrm{SH}), 1.65-1.43\left(\mathrm{~m}, 5 \mathrm{H}, \mathrm{CH}_{2}\right.$ and $\left.\mathrm{CH}_{3}\right), 1.87(\mathrm{~m} .2 \mathrm{H}$, $\mathrm{CH}_{2}$ ), 2.52 (q. $\left.J=7.38 \mathrm{~Hz} .2 \mathrm{H} . \mathrm{CH}_{2}\right), 4.19$ (t. $J=7.50 \mathrm{~Hz}$, $\left.2 \mathrm{H} . \mathrm{CH}_{3}\right) .4 .28\left(\mathrm{~m} . J=7.35 \mathrm{~Hz}, 2 \mathrm{H} . \mathrm{CH}_{2}\right), 7.34(\mathrm{t}, J=1.71$ Hz. lH. Imid), 7.42 (t, $J=1.71 \mathrm{~Hz}$. IH. Imid), $8.90(\mathrm{~s}, 1 \mathrm{H}$. 
Imid); ${ }^{13} \mathrm{C}$ NMR (75.5 MHz. $\left.\mathrm{CDCl}_{3}\right) \delta 15.20 .26 .18 .28 .76$. $28.89,29.04$. 29.10, 29.29, 29.38, 29.43, 29.45, 30.06 . $30.64,45.27,50.14,121.83 .122 .01 .135 .66$.

4d: ${ }^{1} \mathrm{H}$ NMR $\left(300 \mathrm{MHz}, \mathrm{CDCl}_{3}\right) \delta 0.97(\mathrm{t}, J=7.33 \mathrm{~Hz}$. $\left.3 \mathrm{H}, \mathrm{CH}_{3}\right), 1.39-1.26\left(\mathrm{~m}, 21 \mathrm{H}, \mathrm{CH}_{2}\right.$ and $\left.\mathrm{SH}\right), 1.58(\mathrm{~m}, 2 \mathrm{H}$. $\left.\mathrm{CH}_{2}\right), 1.88\left(\mathrm{~m} .2 \mathrm{H}, \mathrm{CH}_{2}\right) .2 .52\left(\mathrm{q}, J=7.31 \mathrm{~Hz}, 2 \mathrm{H} . \mathrm{CH}_{2}\right.$ ). 4.22 (n. + H. $\mathrm{CH}_{2}$ ), 7.25 (s. IH. Inid), 7.27 (s, 1H, Inid). 9.02 (s, lH, Inid); ${ }^{13} \mathrm{C}$ NMR (75.5 MHz. $\left.\mathrm{CDCl}_{j}\right) \delta 19.30$. 26.11. 28.71, 28.85. 29.00. 29.06, 29.26. 29.34, 29.40. $30.06,30.57,31.91,49.77,50.05,122.17,122.32 .135 .77$.

4e: ${ }^{1} \mathrm{H}$ NMR $\left(300 \mathrm{MHz}, \mathrm{CDCl}_{3}\right) \delta 0.89$ (t. $J=6.87 \mathrm{~Hz} .3 \mathrm{H}$. $\left.\mathrm{CH}_{3}\right) .1 .37-1.25\left(\mathrm{~m}, 23 \mathrm{H} . \mathrm{CH}_{2}\right.$ and $\left.\mathrm{SH}\right) .1 .54\left(\mathrm{~m} .2 \mathrm{H} . \mathrm{CH}_{2}\right)$, $1.89\left(\mathrm{~m} .2 \mathrm{H} . \mathrm{CH}_{2}\right), 2.60\left(\mathrm{t}, J=7.32 \mathrm{~Hz}, 2 \mathrm{H}, \mathrm{CH}_{2}\right), 4.24$ (t. $J=$ $\left.7.32 \mathrm{~Hz}, 4 \mathrm{H}, \mathrm{CH}_{2}\right) .7 .40$ (s, 1H. Imid), 7.42 (s, IH, Inid). 9.26 (s, 1H, Inid); ${ }^{13} \mathrm{C} \mathrm{NMR}\left(\mathrm{CDCl}_{3}\right) \delta 13.78,21.98,26.22 .28 .19$, $28.97,29.02 .29 .17,29.23 .29 .39,29.48 .29 .80,30.03,30.18$. $31.00,50.10 .55 .73,122.19 .122 .25 .136 .02$.

4f: ${ }^{1} \mathrm{H}$ NMR $\left(300 \mathrm{MHz}_{2} \mathrm{CDCl}_{3}\right) \delta 0.88(\mathrm{t} . J=6.72 \mathrm{~Hz} .3 \mathrm{H}$. $\left.\mathrm{CH}_{3}\right) .1 .36-1.26\left(\mathrm{~m} .24 \mathrm{H}, \mathrm{CH}_{2}\right.$ and $\left.\mathrm{SH}\right), 1.60\left(\mathrm{~m}, 2 \mathrm{H} . \mathrm{CH}_{2}\right)$. $1.87\left(\mathrm{~m}_{3}, 4 \mathrm{H}, \mathrm{CH}_{2}\right), 2.52\left(\mathrm{t} . J=7.30 \mathrm{~Hz} .2 \mathrm{H}, \mathrm{CH}_{2}\right) .4 .22(\mathrm{q} . J$ $=7.42 \mathrm{~Hz} .4 \mathrm{H}, \mathrm{CH}_{2}$ ), 7.27 (s, 1H, Imid), 7.28 (s, IH. Imid). 9.03 (s, 1H, Inid); ${ }^{13} \mathrm{C} \mathrm{NMR}\left(\mathrm{CDCl}_{3}\right) \delta 13.82 .22 .60 .24 .65$. $25.74,26.11 .28 .31,28.84,29.03 .29 .27,29.44,30.07$. $30.93,34.01,50.27,122.08 .135 .24$

$5 \mathrm{a}:{ }^{1} \mathrm{H}$ NMR $\left(\mathrm{CDCl}_{j}\right) \delta 1.38-1.25(\mathrm{ml} .17 \mathrm{H}) .1 .60(\mathrm{~m}, 2 \mathrm{H})$. $1.86(\mathrm{~m}, 2 \mathrm{H}) .2 .52(\mathrm{q}, J=7.37 \mathrm{~Hz} .2 \mathrm{H}), 3.90(\mathrm{~s} .3 \mathrm{H}), 4.13(\mathrm{t}$. $J=7.49 \mathrm{~Hz}, 2 \mathrm{H}) .7 .24$ (s. 1H), $7.28(\mathrm{~s}, \mathrm{IH}) .8 .49$ (s. $1 \mathrm{H}),{ }^{13} \mathrm{C}$ NMR $\left(\mathrm{CDCl}_{3}\right) \delta$ 24.6. 26.1. 28.3. 28.9, 29.0, 29.3, 29.4. $29.9,34.0 .36 .2,50.1 .122 .0 .123 .6,135.6$.

5b: ${ }^{1} \mathrm{H}$ NMR $\left(300 \mathrm{MHz}, \mathrm{CDCl}_{3}\right) \delta 0.95(\mathrm{t}, J=7.41 \mathrm{~Hz}$. $\left.3 \mathrm{H}, \mathrm{CH}_{3}\right), 1.32-1.2 \mathrm{l}\left(\mathrm{m}, \mathrm{I} 6 \mathrm{H}, \mathrm{CH}_{2}\right.$ and $\left.\mathrm{SH}\right), 1.64(\mathrm{~m}, 2 \mathrm{H}$. $\mathrm{CH}_{2}$ ). 1.91 (n. $\left.2 \mathrm{H} . \mathrm{CH}_{2}\right), 2.68\left(\mathrm{t}, J=7.32 \mathrm{~Hz}, 2 \mathrm{H}, \mathrm{CH}_{2}\right.$ ). 4.20 (m. $4 \mathrm{H} . \mathrm{CH}_{2}$ ), 7.25 (s. IH. Inid). 7.26 (s, 1H, Inid). 9.07 (s, lH, Inid); ${ }^{13} \mathrm{C} \mathrm{NMR}\left(75.5 \mathrm{MHz} . \mathrm{CDCl}_{j}\right) \delta 23.48$. $26.07,28.76 .28 .80,29.03 .29 .11,29.22 .29 .36,29.39$. $29.46,30.09,30.61,50.25,51.62,122.14,122.20$. 135.68.

5c: ${ }^{1} \mathrm{H}$ NMR $\left(300 \mathrm{MHz}_{2} \mathrm{CDCl}_{3}\right) \delta 1.36-1.26\left(\mathrm{nl} .19 \mathrm{H}, \mathrm{CH}_{2}\right.$ and $\mathrm{SH}$ ). $1.65-1.52$ (n. $5 \mathrm{H} . \mathrm{CH}_{2}$ and $\mathrm{CH}_{3}$ ). 1.87 (n. $2 \mathrm{H}$. $\left.\mathrm{CH}_{2}\right) .2 .5 \mathrm{l}\left(\mathrm{q}, J=7.20 \mathrm{~Hz} .2 \mathrm{H} . \mathrm{CH}_{2}\right) .4 .15(\mathrm{t} . J=7.56 \mathrm{~Hz}$. $2 \mathrm{H}, \mathrm{CH}_{3}$ ), 4.25 (q. $J=7.32 \mathrm{~Hz} .2 \mathrm{H}, \mathrm{CH}_{2}$ ). 7.29 (s. IH. Inid). 7.30 (s, 1H, Imid). 8.64 (s. IH. Inid): ${ }^{13} \mathrm{C}$ NMR $(75.5 \mathrm{MHz}$. $\left.\mathrm{CDCl}_{3}\right) \delta 15.09,24.64,26.08,28.20,28.80 .28 .90 .29 .19$. $29.34,29.98 .34 .67,45.11,49.98,50.18 .121 .88 .122 .06$. 135.60 .

5d: ${ }^{1} \mathrm{H}$ NMR $\left(300 \mathrm{MHz} \mathrm{CDCl}_{\mathfrak{j}}\right) \delta 0.95$ (t. $J=7.34 \mathrm{~Hz}$. $\left.3 \mathrm{H}, \mathrm{CH}_{3}\right), 1.39-1.24\left(\mathrm{~m}, 21 \mathrm{H}, \mathrm{CH}_{2}\right.$ and $\left.\mathrm{SH}\right), 1.56(\mathrm{~m}, 2 \mathrm{H}$. $\left.\mathrm{CH}_{2}\right), 1.84\left(\mathrm{ml} .2 \mathrm{H}, \mathrm{CH}_{2}\right) .2 .86\left(\mathrm{t}, J=7.32 \mathrm{~Hz} .2 \mathrm{H} . \mathrm{CH}_{2}\right)$. $4.17\left(\mathrm{~m}, 4 \mathrm{H}, \mathrm{CH}_{2}\right) .7 .33(\mathrm{t}, J=1.65 \mathrm{~Hz} . \mathrm{lH} . \mathrm{Imid}), 7.35(\mathrm{t}, J$ $=1.65 \mathrm{~Hz} .1 \mathrm{H}$. Imid), 8.57 (s. $1 \mathrm{H}$. Inid): ${ }^{13} \mathrm{C}$ NMR $(75.5$ $\left.\mathrm{MHz} . \mathrm{CDCl}_{3}\right) \delta 19.26 .26 .07,28.71,28.81 .29 .00,29.06$. $29.23,29.34$. 29.40, 29.91, 30.58, 31.76, 49.81, 50.09. 122.20. $122.32,135.0$.

5e: ${ }^{1} \mathrm{H}$ NMR $\left(300 \mathrm{MHz}_{2} \mathrm{CDCl}_{3}\right) \delta 0.88(\mathrm{t}, J=6.87 \mathrm{~Hz}$. $\left.3 \mathrm{H}, \mathrm{CH}_{3}\right), 1.4 \mathrm{l}-\mathrm{L} .26\left(\mathrm{~m}, 23 \mathrm{H}, \mathrm{CH}_{2}\right.$ and $\left.\mathrm{SH}\right), 1.62(\mathrm{~m}, 2 \mathrm{H}$. $\left.\mathrm{CH}_{2}\right) .1 .88$ (n. $\left.2 \mathrm{H} . \mathrm{CH}_{2}\right), 2.60\left(\mathrm{t}, J=7.32 \mathrm{~Hz}, 2 \mathrm{H}, \mathrm{CH}_{2}\right.$ ). 4.17 (n. + H. CH $\left.\mathrm{CH}_{2}\right), 7.30$ (s. IH. Inid). 7.36 (s, 1H, Imid). 8.65 (s, lH, Inid); ${ }^{13} \mathrm{C} \mathrm{NMR}\left(75.5 \mathrm{MHz} . \mathrm{CDCl}_{j}\right) \delta 13.84$.
$22.30,24.62,25.73 .26 .10 .28 .30,28.85 .29 .00 .29 .21$ $29.43,29.80 .29 .96 .30 .82 .34 .11 .50 .17 .122 .15,135.25$.

5f: ${ }^{1} \mathrm{H} \mathrm{NMR}\left(300 \mathrm{MHz}, \mathrm{CDCl}_{2}\right) \delta 0.87(\mathrm{t}, J=6.72 \mathrm{~Hz} .3 \mathrm{H}$, $\left.\mathrm{CH}_{2}\right), 1.35-1.25\left(\mathrm{~m}, 24 \mathrm{H}, \mathrm{CH}_{2}\right.$ and $\left.\mathrm{SH}\right), 1.60\left(\mathrm{~m}, 2 \mathrm{H}, \mathrm{CH}_{2}\right)$, $1.86\left(\mathrm{~m}_{3} 4 \mathrm{H}, \mathrm{CH}_{2}\right) .2 .52\left(\mathrm{q}, J=7.35 \mathrm{~Hz} .2 \mathrm{H} . \mathrm{CH}_{2}\right) .4 .17(\mathrm{~m}$, 4H. $\mathrm{CH}_{2}$ ). 7.29 (s, IH. Imid). 7.30 (s, IH. Imid), $8.6 \mathrm{l}$ (s. lH, Inid): ${ }^{12} \mathrm{C}$ NMR $\left(75.5 \mathrm{MHz}, \mathrm{CDCl}_{2}\right) \delta 13.84,22.29,24.62$, $25.73,26.13,28.31$. 28.86. 29.01, 29.29. 29.42. 29.88, $29.96,30.82 .34 .00 .50 .17 .122 .15,135.27$.

6a: ${ }^{1} \mathrm{H}$ NMR $\left(\mathrm{CDCl}_{3}\right) \delta 1.38-1.25$ (m. 17H). 1.60 (m. 2H), $1.86(\mathrm{ml} .2 \mathrm{H}), 2.52(\mathrm{q} . J=7.37 \mathrm{~Hz}, 2 \mathrm{H}) .3 .90(\mathrm{~s} .3 \mathrm{H}), 4.13(\mathrm{t}$. $J=7.49 \mathrm{~Hz} .2 \mathrm{H}), 7.27(\mathrm{~s}, \mathrm{lH}), 7.26(\mathrm{~s} . \mathrm{lH}) .8 .8 \mathrm{l}(\mathrm{s}, \mathrm{lH}) ;{ }^{13} \mathrm{C}$ NMR $\left(\mathrm{CDCl}_{3}\right) \delta 24.6,26.1,28.3 .28 .9 .29 .0 .29 .3,29.4$, 29.9. 34.0, 36.2.50.1, 121.8, 123.5. 135.8.

6b: ${ }^{1} \mathrm{H}$ NMR $\left(300 \mathrm{MHz} . \mathrm{CDCl}_{3}\right) \delta 0.98(\mathrm{t}, J=7.38 \mathrm{~Hz}$ $3 \mathrm{H} . \mathrm{CH}_{2}$ ). $1.36-1.26$ (m. $16 \mathrm{H} . \mathrm{CH}_{2}$ and $\mathrm{SH}$ ). 1.60 (m. $2 \mathrm{H}$, $\left.\mathrm{CH}_{2}\right), 1.91\left(\mathrm{~m}, 2 \mathrm{H}, \mathrm{CH}_{2}\right) .2 .52\left(\mathrm{q}, J=7.26 \mathrm{~Hz}, 2 \mathrm{H}, \mathrm{CH}_{2}\right)$, $4.18\left(\mathrm{~m}, 4 \mathrm{H}, \mathrm{CH}_{2}\right) .7 .26(\mathrm{~s}, \mathrm{lH}$, Imid), $7.28(\mathrm{~s}, 1 \mathrm{H}$, Imid), 8.81 (s. IH. Inid): ${ }^{13} \mathrm{C}$ NMR $\left(\mathrm{CDCl}_{3}\right) \delta$ 19.23. 29.95, 28.64 , $28.67,29.03,29.10$. 29.20. 29.25, 29.39. 29.94. 30.09, $30.52,50.25 .51 .62$. 177.31, 121.56, 122.31. 122.36, 134.94 .

6c: ${ }^{1} \mathrm{H} \mathrm{NMR}\left(300 \mathrm{MHz}, \mathrm{CDCl}_{3}\right) \delta 1.31-1.25\left(\mathrm{~m}, 19 \mathrm{H} . \mathrm{CH}_{2}\right.$ and $\mathrm{SH}), 1.62-1.53\left(\mathrm{~m}, 5 \mathrm{H}, \mathrm{CH}_{2}\right.$ and $\left.\mathrm{CH}_{3}\right), 1.88(\mathrm{~m} .2 \mathrm{H}$, $\left.\mathrm{CH}_{2}\right), 2.52\left(\mathrm{t} . J=7.20 \mathrm{~Hz} .2 \mathrm{H}, \mathrm{CH}_{2}\right), 4.20(\mathrm{t} . J=7.35 \mathrm{~Hz}$, $\left.2 \mathrm{H} . \mathrm{CH}_{3}\right) .4 .28\left(\mathrm{q}, J=7.4 \mathrm{~Hz}, 2 \mathrm{H}, \mathrm{CH}_{2}\right), 7.36$ (t. $J=1.89$ Hz. IH. Imid), 7.43 (t. $J=1.89 \mathrm{~Hz}$. IH. Imid), 8.64 (s, IH. Imid): ${ }^{13} \mathrm{C}$ NMR $\left(\mathrm{CDCl}_{3}\right) \delta$ 13.09. 19.24. 25.96, 28.64 $28.67,28.91,29.10$. 29.20. 29.25, 29.94. 30.52. 31.84, $49.93,50.20 .117 .31 .121 .51$. 122.01, 134.94.

6d: ${ }^{1} \mathrm{H}$ NMR $\left(300 \mathrm{MHz} . \mathrm{CDCl}_{2}\right) \delta 0.95(\mathrm{t}, J=7.34 \mathrm{~Hz}$. $\left.3 \mathrm{H} . \mathrm{CH}_{3}\right), 1.39-1.24$ (m. $21 \mathrm{H} . \mathrm{CH}_{2}$ and $\mathrm{SH}$ ), $1.55 \mathrm{m.} 2 \mathrm{H}$, $\left.\mathrm{CH}_{2}\right), 1.84\left(\mathrm{~m} .2 \mathrm{H}, \mathrm{CH}_{2}\right) .2 .86\left(\mathrm{t}, J=7.32 \mathrm{~Hz}, 2 \mathrm{H} . \mathrm{CH}_{2}\right)$, $4.14\left(\mathrm{~m}, 4 \mathrm{H}, \mathrm{CH}_{2}\right) .7 .32$ (s, lH, Imid), 7.33 (s, lH, Imid), 8.63 (s. IH. Inid): ${ }^{13} \mathrm{C}$ NMR $\left(\mathrm{CDCl}_{3}\right) \delta$ 18.24. 24.96, 28.64 , $28.67,29.10,29.20$. 29.21. 29.25, 29.94. 31.42. 31.85, $49.90,50.10 .122 .56,122.31,122.34,134.64$

6e: ${ }^{1} \mathrm{H}$ NMR $\left(300 \mathrm{MHz}, \mathrm{CDCl}_{3}\right) \delta 0.90(\mathrm{t}, J=6.95 \mathrm{~Hz} .3 \mathrm{H}$, $\left.\mathrm{CH}_{3}\right), 1.34-1.26\left(\mathrm{~m}, 23 \mathrm{H}, \mathrm{CH}_{2}\right.$ and $\left.\mathrm{SH}\right), 1.57\left(\mathrm{~m}, 2 \mathrm{H} . \mathrm{CH}_{2}\right)$, $1.88\left(\mathrm{~m} .4 \mathrm{H}, \mathrm{CH}_{2}\right), 2.6 \mathrm{l}\left(\mathrm{q} . J=7.34 \mathrm{~Hz} .2 \mathrm{H}, \mathrm{CH}_{2}\right), 4.17(\mathrm{~m}$, $4 \mathrm{H} . \mathrm{CH}_{2}$ ). 7.23 (s, IH. Imid). 7.24 (s, IH. Imid), 8.60 (s. $1 \mathrm{H}$, Imid): ${ }^{13} \mathrm{C}$ NMR $\left(\mathrm{CDCl}_{3}\right) \delta$ 19.24. 25.96. 28.64, 28.67 $28.92,29.10,29.20$. 29.21. 29.25, 29.94. 30.42. 31.85, $49.90,50.10 .121 .56,122.31 .122 .35,134.94$

6f: ${ }^{\mathrm{H}} \mathrm{NMR}\left(300 \mathrm{MHz}, \mathrm{CDCl}_{2}\right) \delta 0.95(\mathrm{t} . J=6.72 \mathrm{~Hz} .3 \mathrm{H}$, $\left.\mathrm{CH}_{3}\right), 1.39-1.24\left(\mathrm{~m}, 24 \mathrm{H}, \mathrm{CH}_{2}\right.$ and $\left.\mathrm{SH}\right), 1.55\left(\mathrm{~m}, 2 \mathrm{H} . \mathrm{CH}_{2}\right)$, $1.84\left(\mathrm{~m}_{3} 4 \mathrm{H}, \mathrm{CH}_{2}\right) .2 .86\left(\mathrm{q}, J=7.34 \mathrm{~Hz} .4 \mathrm{H} . \mathrm{CH}_{2}\right) .4 .14(\mathrm{~m}$, $4 \mathrm{H} . \mathrm{CH}_{2}$ ). 7.31 (s, IH. Imid). 7.32 (s, IH. Imid), 8.63 (s. $1 \mathrm{H}$, Imid): ${ }^{13} \mathrm{C}$ NMR $\left(\mathrm{CDCl}_{3}\right) \delta$ 13.09. 19.24. 25.96, 28.64, $28.67,28.91,29.10$. 29.17. 29.20, 29.94. 30.52. 31.84, $49.93,50.20$. 117.31. 121.551, 122.31. 122.36. 134.94.

Preparation of imidazolium ion-terminated selfassembled monolayers (SAMIM-7-10) by spontaneous adsorption of thiol-functionalized ionic liquids 3-6. The Au substrates cleaned prior to adsorption in "piranha" solution ( $25 \%$ peroxide $-75 \%$ sulfuric acid) for 1 min were inmersed in $1 \mathrm{mM}$ solution of thiol terminated ionic liquids 3-6 in absolute ethanol for $3 \mathrm{~h}$. The modified Au surfaces 
were rinsed with ethanol followed by distilled water. and were then dried by blowing with a rapid strean of nitrogen. The surfaces were then characterized by external reflection infrared spectroscopy and contact angle measurements

Acknowledgement. This work was supported by grants from the National Research Laboratory Program and Center for Molecular Design and Synthesis. We thank Professor In Sung S. Choi and Mr. Yong Shik Chi in KAIST for FT-IR measurentents.

\section{References}

1. Ionic Liquids in Sinthesis: Wasserscheid, P. Welton. T.. Eds: Wiley-VCH Verlag: Weinheim. 2003.

2. Ionic Liquids: Indushial Applications for Green Chemistr: Roger. R. D.: Seddon. K. R. Eds.: ACS Symposium Series 818. American Chemical Societ: Washington DC, 2002.

3. Bonhôte P: Dias, A.-P.: Papageorgiou, N.: Kalyanasundaram. K: Grätzel. M. Inorg. Chem. 1996. 35. 1168.

4. Swatloski. R. P.: Visser. A. E.: Matthew Reichert. W: Broker. G. A.: Farina. L. M.: Holbrey, J. D.: Rogers. R. D. Chem. Commu. 2001. 2070

5. Swatloski. R. P.: Visser. A. E.: Matthew Reichert. W: Broker G. A.: Farina. L. M.: Holbrey, I. D.: Rogers, R. D. Green Chent. $2002+81$

6. Dupent. J.: de Souza. R. F.: Suarez. P. A. Z. Chem. Rev: 2002. 102. 3667 .

7. Swatloski. R. P. Spear. S. K.: Holbrey. J. D.: Rogers. R. D. J. Am. Chem. Soc. 2002. 124. 4974

8. Kimizuka, N. Nakashima, T. Langnuir 2001. 17.6759

9. Ho, H. A.: Leclerc, M. J. Am. Chem. Soc. 2003. 125.4412.

10. Dupont. J.: Fonseca. G. S.: Umpierre. A. P.: Fichtner. P. F. P.: Teiseira. S. R. J.Am. Ghem. Soc. 2002. 124. 4228.

11. Zhu. Y.-J.: Wang. W.W. Qi. R.-J.: Hu. X.-L. Angew: Chem. Int. Ed. 2004.13 .1410

12. Huang J.: Jiang T.: Gao. H.: Han, B.: Lil. Z.: Wu. W: Chang, Y.: Zhao, G. Angew Chent Int Ed 2004. 13, 1397.

13. Tivapiboonchaiya C.: Pringle. J. M.: MacFarlane. D. R.: Forsyth. M.: Sun. J Macromol. Chem. Phus. 2003. 204. 2147.

14. Tivapiloonchaiva. C.: Pringle. J. M.: Sun. J.: Bvme. N.: Howlett. P. C. MacFarlane. D. R.: Forsy th. M. Nature 1 faterials 2004. 3. 29.

15. Stathatos. E: Lianos. P: Zakeeruddi, S. M.; Liska, P.: Grätzel, M Chem. Mater 2003. 15, 1825 .

16. UIman. A. Chem. Rev: 1996,96.1533.

17. Kingshott. P.: Griesser. H. J. Gurf. Opin. Solid State Whater Sci. 1999. f. 403

18. Mrksich M. Curr. Opin. Chem. Biol. 2002. 6. 794
19. Whitesides. G. M. Angew: Chem. Int Ed. 1989.28.506.

20. Whitesides. G. M.: Laibinis. P. E. Langmuir 1990. 6.87.

21. Chaudhury, M. K. Whitesides, G. M. Science 1992. 256. 1539

22. Colorado. R.. Jr.: Lee. T. R. Langmiti 2003. 19. 3288

23. Abbott. S.; Ralston. J; Reynolds. G.; Hayes. R. Lamgnnir 1999 15. 8923 .

24. Ichimura. K.: Oh. S.-K.: Nakagawa. M. Science 2000. 288. 1624

25. Lahan11. , T: Mitragotri. S.: Tran. T.-N.: Kaido. H.: Sundaram1. T. Choi. I. S.; Hoffer. S.: Somorjai. G. A.: Langer, R. Science $\mathbf{2 0 0 3}$ 299.371.

26. Abbott. N. L.: Kumar. A.: Whitesides, G. M. Chen Mater, 1994 6.596.

27. Itoh. M.: Nishihara. H.: Aramaki. K. J. Electrochem. Soc. 1994. 1H1.2018.

28. Sinapi. F.: Forget, L:- Delhalle. J:- Mekhalif. Z. Appl. Sinf. Sci. 2003. 212,464

29. López. G. P.: Albers, M. W.: Schreiber, S. L.: Carroll. R.; Peralta. E.: Whitesides. G. M. 1993. 115.5877.

30. Mrksich. M.: Whitesides. G. M. Anm. Rev Bioplns. Biomol Struct. 1996. 25.55

31. Mrksich. M. Cell Mol. Life Sci. 1998, 51.653.

32. Kingshott, P.: Griesser. H. J. Cum Opin. Solid State Matet Sci. 1999. 4.403 .

33. Mrksich. M. Cur. Opin. Chem. Biol 2002. 6.794

34. Schaeferling. M.: Schiller. S.: Paul. H.: Kruschina. M. Pailichova. P.: Meerkamp. M: Giammasi. C.: Kambhampati. D Electrophoresis 2002. 23,3097.

35. Minko. S.: Muller, M.: Motornov, M.: Nitschke. M.; Grundke, K.; Stamm. M. J.Am. Chem. Soc. 2003. 125. 3896.

36. Julthongpiput. D.: Lin. Y.-H.: Teng. J.: Zubarew. E. R.: Tsukruk: V V. Langmair 2003. 19.7832

37. Julthongpiput. D.: Lin. Y.-H.: Teng. J.: Zubarev. E. R.: Tsukruk. V V. J Am Chem Soc 2003, 125, 15912

38. Chatelier, R. C. Drummond. C. J.; Chan. D. Y. C.; Vasic. Z. R. Gengenbach. T. R: Griesser, H. J. Langmii 1995, 11,4122.

39. Ista. L. K.: Perez-Luna. V. H.: Lopez. G. P. Appl. Eniron. Ificrobiol 1999.65. 1603 .

40. Nath. N.: Chilkoti. A. Adv. Mater: 2002. 14. 1243.

41. de Crevoisier. G;: Fabre, P; Corpart, J.-M.: Leibler. L. Science 1999. 285,1246 .

42. Ohnishi. S.: Ishida, T.: Yaminsky. V. V: Christenson. H. K Langmuir 2000. 16. 2722

43. Gallardo. B. S.: Gupta. V. K.: Eagetton1. F. D.: Tong. L. I.: Craig. V. S.: Shah. R. R.: Abbott. N. L. Science 1999. 283.57

4. Lee. B. S. Chi, Y. S.; Lee. J. K: Choi, I. S.: Song. C. E. Namgoong. S. K. Lee, S.g. J. Am. Chent Soc 2004, 126.480

45. Chi. Y. S.; Lee. J. K.; Lee, S.-g: Choi. I. S. Lamgnimir 2004. 20. 3024

46. Itoh. H.: Naka. K.: Chujo. Y. J. Am. Chem. Soc. 2004. 126. 3026.

47. Porter. M. D.: Bright. T. B.: Allara. D. L.: Chidsey. C. E. D. J. Am. Chent Soc. $1987,109.3559$. 